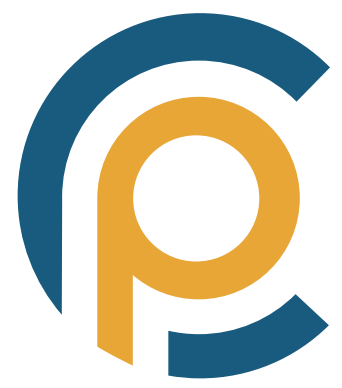

\title{
La Educación de Personas Adultas a los Cincuenta Años de la Ley General de Educación
}

\author{
Adult Education on the Fiftieth Anniversary \\ of the General Education Law
}

Recibido: 21/05/2020 | Revisado: 02/09/2020 | Aceptado: 28/09/2020 | Publicado: 22/12/2020

\section{Óscar Medina Fernández}

Universidad de Las Palmas de Gran Canaria (España)

oscar.medina@ulpgc.es

https://orcid.org/0000-0003-3167-5038

Resumen: Utilizando como referencia la Ley General de Educación de 1970, así como otras leyes educativas españolas anteriores y posteriores, se aborda la evolución que ha experimentado la educación de personas adultas desde el siglo XIX hasta la actualidad, analizando tres grandes desafíos que consideramos imprescindibles para la implantación de una política educativa creíble y viable en este campo: a) Oferta educativa específica versus carácter compensatorio y sustitutorio; b) Pedagogía diferenciada versus modelo escolar; y c) Legislación sobre el universo educativo versus sistema educativo formal y oficial. Dicho análisis, en el que se contrasta la legislación con las realizaciones educativas y con las teorizaciones de autores, nos lleva a concluir que, a lo largo ya de más de dos siglos de existencia, en España no ha habido política educativa alguna en materia de educación de las personas adultas. $Y$ todo ello por varias razones: porque se desconoce el crecimiento que ha experimentado este sector educativo, ignorando asimismo los beneficios personales, culturales, económicos, sociales y políticos que tiene esta realidad; porque las leyes se han limitado a regular el sistema educativo formal y oficial, dejando fuera buena parte de la educación que tienen y realizan las personas adultas; y porque la concepción pedagógica que lo justifica no ha trascendido a la sociedad ni a las instituciones competentes en la materia.

Palabras clave: educación de adultos, nueva oportunidad educativa, formación continua, educación no-formal, educación informal.
Abstract: Using as a reference the 1970 General Education Act, as well as other earlier and later education laws, I address the evolution of adult education from the 19th century to the present day, exploring three great challenges essential for the implementation of a credible and viable educational policy: a) Specific educational offer versus compensatory and substitute character; b) Differentiated pedagogy versus school model; and c) Legislation on the educational universe versus formal and official educational system. This analysis, in which legislation is contrasted with educational achievements and with the theories of authors, leads us to conclude that, throughout more than two centuries of existence, in Spain there has been no educational policy whatsoever in the area of adult education. There are several reasons for this fact: because the growth that this educational sector has experienced is unknown, and the personal, cultural, economic, social, and political benefits of this reality are not known; because the laws have been limited to the regulation of the formal and official educational system, leaving out a good part of the education that adults have and carry out; and because the pedagogical concept that justifies it has not transcended society or the competent institutions in the field.

Keywords: adult education, continuation of education, further training, non-formal education, informal education. 
Han transcurrido cincuenta años desde la aprobación de la LGE (Ley General de Educación) en 1970. A lo largo de este medio siglo ¿qué ha sucedido con la educación de personas adultas en España? En este artículo se analizan algunos antecedentes y lo que significó la LGE, abordando también su evolución hasta nuestros días. Más concretamente, nos ceñimos a las dos preguntas siguientes que, entre otras, se formulan en la presentación del monográfico: ¿Cómo ha cambiado la educación de personas adultas en estos cincuenta años? ¿Qué cambios ha experimentado la educación no formal e informal en este período? Dos preguntas que, como veremos a lo largo de este trabajo, están estrechamente relacionadas entre sí.

Si contrastamos la legislación con las realizaciones educativas y con las teorizaciones de autores, la conclusión no puede ser otra que la siguiente: a lo largo ya de más de dos siglos de su existencia, la educación de las personas adultas no ha contado en España con una política educativa mínimamente creíble y viable. La razón tiene que ver con el modo en que se han abordado los siguientes tres grandes dilemas, que han condicionado su desarrollo en determinados momentos claves de su historia: a) ¿La oferta educativa es específica y tiene valor en sí misma o por el contrario se desliza por una concepción meramente compensatoria? b) ¿Las prácticas educativas se realizan de forma diferenciada con una Pedagogía específica o se mimetiza lo que se hace en las escuelas para los infantes? c) ¿Las leyes de educación se ocupan del universo educativo, es decir, de la educación de la sociedad, incluyendo la educación de las personas adultas, o solo se centran en las enseñanzas formales y oficiales del sistema educativo, dejando fuera otras formas de educación?

A continuación, teniendo como referencia la LGE, abordamos las tres preguntas anteriores, analizando la contribución de las principales leyes educativas españolas y con ello la evolución de la educación de personas adultas a lo largo de su historia.

\section{Oferta educativa específica versus carácter compensatorio y sustitutorio}

Tradicionalmente la educación de personas adultas no ha sido considerada por las leyes educativas como un tipo de educación necesaria y con valor propio; más bien lo que ha sucedido es que la legislación anterior a la LGE ha apostado por un perfil compensatorio que incluso se reforzaba con la consideración de sustitutorio. Nos referimos a que, lejos de concebirla como una oferta educativa que tiene valor en sí misma, como fenómeno optimizante en términos personales y sociales, independientemente de la evolución de otros sectores educativos y de la instrucción previa que posea la población adulta, lo que se busca es que las personas adultas tengan una segunda oportunidad y puedan poner remedio al hecho de no haber estudiado por razones de trabajo, ser analfabetos o haber olvidado lo aprendido. Este es el sentido remedial y paliativo que tiene la educación de la población adulta, a juzgar por lo establecido en las primeras leyes educativas. Las casi inexistentes referencias legales a esta educación en las leyes del siglo XIX y parte del siglo XX abundan en lo que decimos.

Durante el primer tercio del siglo XIX se publica la Ley Provisional de Instrucción Primaria de 1838 para llevar a la práctica el ideario liberal en materia de educación, estableciendo las primeras bases normativas de la enseñanza. Todo el texto se centra en la instrucción de los "niños", a los que se les considera destinatarios de la ley, dedicando solo una línea a las "escuelas de adultos": "Así mismo, procurará el gobierno la conservación y fomento de las escuelas de adultos" (art. 37). Décadas después se aprueba la Ley de Instrucción Pública de 1857 para completar la organización de la enseñanza, ya con tres niveles, y para ordenar mejor el gobierno y la administración de la instrucción. También en este caso la ley se ocupa de la instrucción de los menores como únicos destinatarios, salvo un artículo para los "adultos", donde queda más claro su carácter compensatorio: "Igualmente fomentará el establecimiento de lecciones de noche o de domingo para los adultos cuya instrucción haya sido descuidada, o que quieran adelantar en conocimientos" (art. 106). Casi a mitad del siglo XX se aprueba la Ley de Educación Primaria de 1945, que establece lo que va a ser la educación durante el franquismo. Como en las leyes anteriores, también aquí el contenido se centra en la educación de los "niños", dedicando a la "educación de adultos" solo dos artículos: sobre las "escuelas de adultos" y sobre las "misiones pedagógicas" (arts. 31 y 32). 
Se trata de una época en la que, con relación a la educación de personas adultas, la preocupación principal de las políticas educativas se centraba en la alfabetización, debido a dos razones: la creciente industrialización unido al incremento de las grandes ciudades y las insuficiencias de la instrucción de la infancia. Tan estrecha era la relación entre alfabetización de adultos y déficit de formación en la infancia, que se pensaba que la realización efectiva de la escolaridad infantil acabaría haciendo innecesarias las "escuelas de adultos" que podrían desaparecer, acentuando así la concepción compensatoria con este carácter sustitutorio (Guereña, 1992; Gómez R. de Castro, 1995). Dicho de otra manera, se abren escuelas de adultos porque no funcionaban bien las escuelas de los niños y sucedía con mucha frecuencia que cuando estos ya eran adultos se comprobaba que o no habían ido a la escuela (porque no había o por razones de trabajo), o habían olvidado lo aprendido (por falta de práctica), o volvían a ser analfabetos (porque no cultivaban la lectoescritura). Esto es lo que significa compensatorio, dado que la función de las escuelas de adultos era compensar, remediar, dar una segunda oportunidad a los adultos, dado que perdieron la primera oportunidad cuando eran niños.

Así, para paliar las secuelas tan negativas de las políticas educativas para la infancia y para responder a las demandas de formación que exigía la Revolución Industrial y los nuevos valores asociados a la Revolución Francesa y la llustración, el proceso de alfabetización de la población adulta en este período se lleva a cabo a través de una doble vía: por un lado, en las "escuelas de adultos" promovidas por el Estado, con clases nocturnas y durante los domingos; y por otra parte, mediante la iniciativa social que desarrolla programas de educación popular, formación laboral y alfabetización a cargo de asociaciones, grupos políticos, universidades, la Iglesia, etc., (Tiana Ferrer, 1991; Moreno Martínez, 1992b; Delgado Granados, 2005).

Sin embargo, con la Ley General de Educación de 1970 se da un giro en esta dirección. Es una ley que nace con una vocación renovadora en un momento histórico en el que se suceden cambios educativos relevantes a nivel internacional, como el movimiento pedagógico de la Escuela Nueva, las primeras publicaciones sobre educación permanente, la pedagogía de la liberación de Paulo Freire, las experiencias de educación no directiva y la influencia de la UNESCO, ya con 25 años desde su creación en el seno de la ONU (Organización de las Naciones Unidas). Ello explica que con esta ley se aleje definitivamente a la educación de personas adultas del carácter compensatorio, superando también la consideración de oferta sustitutoria. Prueba de ello es el hecho de incluir el nivel de "Educación Permanente de Adultos" en la estructura del sistema educativo (una decisión controvertida, pero que en su momento tuvo ventajas en términos de reconocimiento de una oferta con valor propio), vinculando la educación al principio de educación permanente.

A partir de ahora vemos que la educación de personas adultas se normaliza institucionalmente y adquiere valor en sí misma como una oferta educativa que no se vincula a la formación previa que se posea, ni depende de la evolución efectiva de la escolaridad infantil. Algunos párrafos legales confirman esta posición: se busca "dar plena efectividad al derecho de toda persona humana a la educación"; "hacer partícipe de la educación a toda la población española"; "ofrecer a todos la igualdad de oportunidades educativas"; construir "un sistema educativo permanente [...], capaz de desarrollar hasta el máximo la capacidad de todos y cada uno de los españoles"; asegurar "la unidad del proceso de la educación" en la idea de que "la educación es tarea de todo el país" (preámbulo y art. 9.1).

Durante 20 años está vigente la LGE hasta que, en 1990, se aprueba la Ley Orgánica de Ordenación General del Sistema Educativo, la LOGSE, entre otras razones, para adaptar el sistema educativo a la Constitución de 1978 (que crea un orden social y político democrático, así como una organización territorial descentralizada) y para ajustar la realidad educativa española a la Unión Europea en la que España se había integrado desde 1986. Así se explica que con esta ley se produzcan cambios importantes a nivel general y también sobre la educación de personas adultas, cuyo tratamiento todavía se aparta más del tradicional enfoque compensatorio y sustitutorio. Dos son los argumentos principales que acreditan su carácter propio en esta ley frente a los planteamientos tradicionales: por una parte, la decisión de no incluir la educación de personas adultas en la estructura del sistema educativo (lo cual resulta 
paradójico, comparado con lo establecido por la LGE), reconociendo así la existencia de todo un sector o ámbito de la educación ya con entidad propia, cuyos destinatarios son las personas adultas; y por otra parte, el refuerzo del principio de educación permanente (también establecido en la LGE) en el marco más general del derecho de todos a la educación y a la igualdad de oportunidades. No pocos textos de la ley lo demuestran: "la educación será permanente, y así lo proclama la ley al determinar que ese será el principio básico del sistema educativo" (preámbulo); "todos tienen derecho a la educación" (art. 27.1); "el derecho a la educación es un derecho de carácter social" (preámbulo); lo que se quiere hacer con la ley es "una reforma profunda del sistema educativo" (preámbulo); "el sistema educativo tiene que responder a las aspiraciones educativas de la sociedad" y contribuir "a la reducción de la injusta desigualdad social" (preámbulo), etc.

Por tanto, debemos felicitarnos del carácter propio que por fin acaba teniendo la educación de personas adultas a partir de la LGE y la LOGSE, como una oferta educativa específica y optimizante por sí misma, como otros campos de la educación, pero que sigue transitando por esa doble vía de la que hablábamos antes.

Una de esas vías, promovida por el Estado mediante leyes que regulan el sistema educativo, es la que se centra en la formación básica de la población adulta, que tiene lugar en las escuelas y/o centros de personas adultas; vía esta que inicia su camino desde un lugar aparte, la escuela, que, desvinculada de la comunidad y sin relación con los problemas cotidianos, laborales, culturales y sociales del alumnado, acaba configurándose como oferta oficial de carácter formal.

La segunda vía, mediante la iniciativa social y privada y al margen de la legislación y del sistema educativo, interviene con programas y experiencias de educación popular para personas adultas en centros cívicos, casas de la cultura, centros municipales, escuelas populares, programas de extensión universitaria, ateneos, gabinetes y universidades populares. Considerada esta vía como una oferta de carácter no formal e informal, su nacimiento obedece a varias razones que explican sus finalidades: para suplir las deficiencias de la vía formal que operaba de espaldas a la realidad, para reforzar el proceso de alfabetización y culturalización de la población con independencia de los requisitos formales y para ofertar la educación y la cultura a todos los ciudadanos y en los lugares más necesitados.

Doble vía, cuya relación entre sí ha sido diferente dependiendo de cada momento histórico y de las ofertas de algunos centros, pero que sigue enfrentándonos a dos problemas que están cortando las alas de este sector educativo, constriñéndolo así a un vuelo rasante: la necesidad de un tratamiento diferenciado y la falta de coordinación entre ofertas e instituciones.

\section{Pedagogía diferenciada versus modelo escolar}

Establecido el carácter propio de la educación de personas adultas, que establece la LGE y refuerza la LOGSE, lo lógico sería que tal sector contara con un tratamiento diferenciado en el sistema educativo, una Pedagogía propia, capaz de responder a las características psicológicas y sociales de los destinatarios, coordinando además las dos vías antes señaladas. Pero esto ha sido así solo en parte: a través de las leyes, algo se ha avanzado en el tratamiento diferenciado de la vía formal, pero nada respecto de su coordinación con la vía no formal e informal.

En realidad, lo que ha sucedido es que, antes de la LGE, la educación de personas adultas ya se caracterizaba por el modelo escolar, entendiendo por ello la fuerte dependencia de la escuela que mantienen las actividades educativas dirigidas a la población adulta. No se educa a las personas adultas de forma diferente a la infancia, sino que se imparte un tipo de educación prácticamente igual a la de los menores en las escuelas, con las únicas diferencias sobre la edad y los horarios (Guereña, 1992; Medina Fernández, 1997; Viñao Frago y Moreno Martínez, 2003; Sáez Carreras, 2007).

Tal mimetismo de la escuela infantil, sutilmente esbozado durante el siglo XIX, se consolida durante casi todo el siglo XX, aplicando también a la educación de personas adultas la Pedagogía Escolar dominante de la época, centrada en la infancia y en la escuela. La norma fundamental de todo este período (base de posteriores órdenes y decretos), que fija el rumbo escolar para la población adulta, 
es el Real Decreto de 4 de octubre de 1906, estableciendo que el objeto de las clases de "adultos" es "ampliar y perfeccionar la educación dada en las escuelas primarias diurnas" (art. 1), donde se impartirán las "enseñanzas de cultura general que forman parte del programa de las escuelas primarias" (art. 16). Incluso en la Ley del 45, se afirma el doble cometido de las "escuelas de adultos": "iniciar o completar la enseñanza primaria y formar o perfeccionar en el orden profesional a aquellos alumnos que ya posean, aunque elementalmente, los conocimientos de la Escuela" (art. 31). Con todo, este mimetismo de la educación infantil y de la escuela solo tiene algunas excepciones en las normas señaladas: las referencias al calendario y los horarios y las pautas metodológicas, que, a todas luces, resultan muy débiles para hablar de una Pedagogía diferenciada.

A partir de la LGE se producen algunos avances para superar el modelo escolar, pero resultan tímidos y claramente exiguos si tenemos en cuenta las normas de aplicación y la práctica de los centros. Varios artículos de esta ley, que se apartan de la concepción tradicionalmente escolarizante, reparan en que las enseñanzas para personas adultas debían ser diferentes a las de la infancia. Se afirma que "el contenido y los métodos educativos de cada nivel se adecuará a la evolución psicobiológica de los alumnos" (art. 9.d), apoyando así una cierta Pedagogía diferenciada, con una organización propia, dotada de "centros específicos", y también "centros ordinarios" (art. 44.1); planteamiento que se acentúa todavía más al afirmar que "la planificación de las actividades de educación permanente de adultos se basará en investigaciones" basadas, entre otras cuestiones, en la Psicología de los adultos y en la Sociología del momento (art. 45.1).

Sin embargo, nuevamente vemos que una cosa es la letra y otra la realidad, como se demuestra en las Orientaciones Pedagógicas del 74 para la EGB (Educación General Básica) de adultos, que traslucen el distanciamiento entre las declaraciones teóricas y las decisiones prácticas, la desconexión con los propios principios de la ley, la resistencia al tratamiento diferenciado de este campo de la educación, o, en suma, la pérdida de una oportunidad para adaptar esta formación a las características y complejidad de la población adulta (Moreno Martínez, 1992; Medina Fernández, 2000; Cabello Martínez, 2002).

Posteriormente, con ocasión de la aprobación de la LOGSE se avanza todavía más para superar el modelo escolar, inicialmente, porque, durante los debates previos a su aprobación, se conocen acuerdos importantes, que se reflejan en el Libro Blanco para la Reforma del Sistema Educativo (MEC, 1989), donde se afirma que la "educación de adultos no puede ser considerada intrínsecamente sino como un subconjunto integrado en un proyecto global de Educación Permanente" (pp.191), razón por la cual "no parece, por lo tanto, adecuado dar a la educación de adultos el mismo tratamiento que al resto del sistema educativo" (pp. 189).

No obstante, la evolución de los acontecimientos y el texto que acaba aprobándose en el parlamento indican que los avances de la LOGSE para establecer una política diferencial en este campo de la educación resultan claramente insuficientes por varias razones. La primera, porque con la aprobación de la LOGSE se desvanecen las expectativas (de los profesionales y del propio Ministerio de Educación) puestas en la aprobación de una ley sobre educación de personas adultas, aprobada en el parlamento nacional para todo el Estado, que consagre su carácter propio y tratamiento diferencial, como se explicaba en el Libro Blanco de la Educación de Adultos en España (MEC, 1986). Y la segunda, porque la ley orgánica de 1990 no recoge toda la complejidad del tratamiento diferenciado y la necesaria coordinación que merecían las dos vías (formal y no formal e informal) de este sector educativo y que también se describía en la obra citada; no en vano son muchos los autores que reconocen la función y extraordinaria acogida del citado Libro Blanco entre los profesores, como una útil herramienta que por fin respondía a las experiencias innovadoras, de acuerdo con las directrices de los organismos internacionales que, apartándose de la concepción escolarizada, optan por un modelo social de educación de personas adultas de amplia base para el desarrollo personal y comunitario (Viladot y Romans, 1988; Jabonero Blanco et al., 1997; Marzo y Figueras, 1990; Viñao Frago y Moreno Martínez, 2003; Sarrate Capdevila y Pérez Guzmán Puya, 2005). 
No podemos pasar por alto que en este Libro Blanco sobre la educación de adultos en España se plantean dos cuestiones decisivas para el desarrollo de este campo de la educación que respondían a las preocupaciones de los profesionales del momento y tenían en cuenta no pocas experiencias innovadoras con un fuerte contenido local y emancipador: a) frente al tradicional modelo escolar, se plantea un nuevo modelo social de educación de personas adultas, como subconjunto de un proyecto global de educación permanente, para la totalidad de los procesos de educación (sean formales o no formales), con mecanismos de coordinación y acreditación entre ofertas y actores intervinientes; y b) frente a un tipo de actividad centrada exclusivamente en el aula, se establece el proyecto de base territorial como la unidad espacial de referencia, lo que nuevamente obliga a la coordinación y la colaboración interinstitucional (MEC, 1986).

Se trata de una doble perspectiva con la que se identifica un número importante de investigadores que optan por una educación de adultos integradora, crítica, social y emancipadora, sea formal, no formal o informal, se refiera a la formación básica o a la educación popular, se imparta en centros oficiales o en cualquier otra institución o contexto (Quintana Cabanas, 1986; Flecha, 1990; Lucio-Villegas Ramos, 1993; Guereña y Tiana Ferrer, 1994; Mezirow, 1994; Formariz Poza, 1995; García Carrasco, 1997; Sáez Carreras, 1998; Camacho Herrera, 1999; Sebastián Vicente, 2001; Trilla Bernet, 2002; Requejo Osorio, 2003; Tiana Ferrer y Sanz Fernández, 2003; Caride Gómez, 2009). Un enfoque socioeducativo que concibe la educación de personas adultas como un ámbito de la educación social, cuyo marco terminológico, teórico, histórico y prescriptivo es la Pedagogía Social.

En su lugar, lo que hace la LOGSE es limitarse al tratamiento diferenciado solo de la vía formal, es decir, de los currículos de las enseñanzas oficiales del sistema educativo (Educación Básica, Bachillerato y Formación Profesional), utilizando expresiones como las siguientes en caso de que los destinatarios sean personas adultas: "contarán con una oferta adaptada a sus condiciones y necesidades" (art. 52.1), "oferta específica y una organización adecuada a sus características" (art. 53.2). Parecía estar claro, a partir de tales artículos y de otros que el currículo de las enseñanzas oficiales, sobre todo el de la educación básica, tenía que ser diferente al del régimen general para niños y adolescentes. Un asunto este que genera no pocas reflexiones y propuestas por parte de algunos autores (Requejo Osorio, 1992; Debón Hernández y Llop Chulvi, 1995), suscitando además un importante debate entre los profesores y la administración educativa (central y autonómica), que reflejaba dos concepciones sobre el currículo de Educación Básica de las personas adultas: por una parte, los defensores de una mera adaptación curricular, y por otra, quienes optaban por un currículo específico y diferente (MEC y CCAA, 1993 y 1995); dos concepciones que se resuelven de forma diferenciada a nivel territorial, como Canarias (Consejería de Educación, Cultura y Deportes, 1992), que opta por una oferta curricular específica, y el Ministerio de Educación (para lo que todavía pertenecía al territorio MEC), que se limita a una adaptación curricular.

Finalmente, hemos de señalar otros cambios relevantes sobre la evolución de la educación de personas adultas en este período, que evidencian nuevos avances pedagógicos, una realidad educativa diferenciada a nivel territorial, así como el papel que han jugado las CCAA (Comunidades Autónomas) en su desarrollo. Cabe destacar en este sentido la evolución que, en términos de Pedagogía diferenciada para las personas adultas, han tenido los sistemas de reconocimiento y acreditación de la experiencia y de las enseñanzas no formales, que partiendo de dispositivos y experiencias realizadas en algunas CCAA (como Cataluña, País Vasco y Canarias), se consolidan legalmente a nivel de todo el Estado, mediante la Ley Orgánica de las Cualificaciones y de la Formación Profesional en el año 2002 y su correspondiente Real Decreto siete años después. Por otra parte, perdida la ocasión de aprobar una ley general sobre educación de adultos a nivel nacional, algunas CCAA, como Andalucía, Aragón, Cataluña, Galicia, Valencia, Castilla León, Castilla La Mancha, Navarra, Canarias, etc. han aprobado en sus propios parlamentos una ley de educación de personas adultas, cuya aproximación a los planteamientos sociales y comunitarios del Libro Blanco será diferente en cada territorio, y cuyo cumplimiento de lo establecido en las propias leyes también será diferente según las CCAA (Sanz Fernández, 2002; Lancho Prudenciano, 2005; Sarrate Capdevila y Pérez de Guzmán Puya, 2005). 
En resumen, podemos concluir que, frente a los enfoques teóricos que nos remiten a un modelo social de educación de personas adultas, capaz de coordinar en cada territorio las dos vías (formación básica y educación popular), la práctica continúa regida por el modelo escolar, aunque más la primera vía que la segunda. Una carencia esta, nada desdeñable, que está cercenando el desarrollo de este campo, debido a la inercia de las autoridades educativas que tradicionalmente han regulado solo las enseñanzas formales y oficiales del sistema educativo.

\section{Universo educativo versus enseñanzas formales y oficiales del sistema educativo}

El tercer envite al que tradicionalmente se ha enfrentado la educación de las personas adultas a lo largo de la historia tiene que ver con la concepción de la educación que subyace a cada una de las leyes. ¿Tales leyes de educación se ocupan del universo educativo, es decir, de la educación de la sociedad, de las personas de todas las edades, o solo de las enseñanzas y de la educación que va dirigida a una parte de la sociedad?

Desde nuestro punto de vista, en general las leyes educativas se han centrado casi exclusivamente en la estructura y organización de las "enseñanzas formales", que cuentan con la acreditación del Estado, destinadas a la infancia, a la juventud y también a algunas personas adultas (las que carecen de titulación oficial). Lo que regulan, pues, las leyes es la formación que se imparte en los diferentes niveles del "sistema educativo" (la primera vía, comentada anteriormente) y que conduce a las titulaciones oficiales en cada momento.

Nada, pues, sobre coordinación, fomento, apoyo, seguimiento y financiación de esa otra vía u oferta de educación popular, destinada también a la población adulta, catalogada como "educación no formal e informal", que imparten otros ministerios del Estado, las fuerzas armadas, los ayuntamientos a nivel municipal, los sindicatos, las universidades, las asociaciones, las universidades populares, etc.; ofertas sociales y privadas de las que hablan no pocos autores (Flecha et al., 1988; Gómez R. de Castro, 1995; Moreno Martínez y Viñao Frago, 1997; Sanz Fernández, 2006), destacando la función que tienen en la extensión de la cultura, la formación laboral, la educación ciudadana y la participación social de las personas adultas.

Esta concepción restringida de la educación es la que ha prevalecido en las primeras leyes educativas españolas. Se regula la instrucción (que más adelante coincidiría con la enseñanza formal y oficial) que han de recibir las jóvenes generaciones y algunos adultos que sean analfabetos o hayan olvidado lo aprendido, dejando fuera las experiencias de educación popular, que llegaron a tener tanto arraigo y repercusión social en esta misma época. No obstante, hemos de señalar una salvedad, a saber, el papel también del Estado para otras actividades educativas de carácter no formal e informal, como las misiones pedagógicas, que cumplían una función importante en las campañas de alfabetización y en el apoyo de la educación popular y cultural que se desarrollaba en las "escuelas de adultos" y en otros centros no oficiales. Creadas en el año 1922, las misiones pedagógicas, que se utilizaron durante la Segunda República, se restablecen durante la dictadura a través de la Ley de 1945, donde queda claro su carácter no formal e informal: "Desarrollarán su actividad mediante bibliotecas circulantes, conferencias, discotecas, exhibiciones teatrales, exposición de reproducciones artísticas, cine educativo, emisiones de radio y otros medios análogos, con preferencia los que contribuyan a mejorar la vida rural" (art. 32).

Más adelante, con la publicación de la LGE no parece que esta situación cambie. Aunque en la ley se hable de educación permanente como un derecho de todos, la identificación entre educación y sistema educativo conduce a que se regule prácticamente lo mismo: el sistema educativo formal y oficial, dejando fuera buena parte de las oportunidades educativas de carácter no formal o informal que realizan sobre todo las personas adultas. De hecho, en la ley se habla de una doble oferta educativa: por un lado, "Educación General Básica, Bachillerato y Formación Profesional", y por otro, "actividades de perfeccionamiento, promoción, actualización y readaptación profesional, así como promoción y extensión cultural” (art. 44.1); pero, en la práctica, lo que se regula y a lo que se destinan medios económicos, recursos y 
profesorado es la Educación General Básica. Lo mismo cabe decir de las Orientaciones Pedagógicas del 74 , centradas, sobre todo, en asegurar que las personas adultas reciban las mismas enseñanzas que la infancia para poder acceder al mismo título. Pruebas evidentes de que la ley se tornó incapaz de salvar buena parte de las inercias del pasado, que ponían de manifiesto el vagón de cola que representaba la educación de personas adultas en el sistema educativo: falta de interés y de orientación de la política educativa, insuficiente preparación específica del profesorado, escasez de recursos, inexistencia de mecanismos de coordinación con otras ofertas educativas (de otros ministerios y organismos públicos, de los ayuntamientos y de la sociedad civil), pero lo más destacable, ausencia también de una cultura pedagógica más acorde con los tiempos (Moreno Martínez, 1992a).

Pasados veinte años, con la aprobación de la LOGSE pasa algo semejante. Pese a que en esta ley se consagra el principio de educación permanente y el derecho de todos a la educación, tratando de reducir las desigualdades, nuevamente la pretensión de regular el sistema educativo revela implícitamente una concepción restringida de la educación y de las personas destinatarias. Esto queda claro cuando la LOGSE define la estructura, componentes y niveles del sistema educativo, limitándose a regular lo que siempre hemos entendido por educación sistemática, oficial o formal, que es la que se regula por parte de las autoridades educativas de un país, y cuyo público objetivo son, precisamente en función de tales niveles, los infantoadolescentes y los jóvenes universitarios.

El resultado es que con la LOGSE se superan algunas limitaciones que tradicionalmente padecía este sector de la educación, pero se deja fuera del sistema educativo buena parte de la educación que realizan las personas adultas: la educación no oficial (no formal), la formación que ofertan las universidades populares, la educación de los trabajadores en las empresas, las actividades educativas que se imparten dependiendo de los ayuntamientos, los programas de animación sociocultural, los proyectos de desarrollo local y comunitario, los procesos de autoformación, las ofertas para mayores de las propias universidades, los centros cívicos, las casas del pueblo, los gabinetes literarios, las tertulias literarias, las comunidades de aprendizaje, etc.; en suma, las diferentes oportunidades de educación no formal e informal.

Todo parece indicar, pues, que la LGE y la LOGSE en determinados textos (como los que se refieren al derecho de todos a la educación, a la regulación del sistema educativo de acuerdo con la educación permanente, a los fines del sistema educativo en términos de educación global e integral de todas las facultades del ser humano, a la reducción de las desigualdades, etc.) acaban dando la espalda a la realidad al no contemplar la educación en su conjunto y diversidad y solo referirse a una parte de la educación del país. La conclusión, por tanto, es que tales textos y artículos de la ley más bien han servido para las declaraciones y las justificaciones, y se han quedado así en la mera retórica.

\section{Conclusiones y propuestas para el debate}

Presentamos para terminar algunas conclusiones y propuestas que sometemos al debate a través de esta revista, considerando dos posibles lecturas del mismo fenómeno: una se refiere al contenido de las leyes, las declaraciones, los artículos, lo que se dice, la literalidad, que puede coincidir o no con las normas y órdenes que a la postre tratan de regular la práctica educativa en los centros; la otra alude a la reflexión, los análisis y la investigación de diferentes autores que, a la luz de experiencias relevantes y prácticas innovadoras, aportan propuestas pedagógicas en el seno de la propia comunidad científica.

En el primer caso, si analizamos las principales leyes y normas educativas españolas, vemos que desde el principio han caracterizado la educación de personas adultas con tres rasgos principales: a) Era un tipo de educación eminentemente compensatoria hasta el extremo de ser considerada sustitutoria; realidad que a partir de la LGE cambia y comienza a tener valor en sí misma. b) La acción educativa desde el principio se ha venido rigiendo por el denominado modelo escolar o Pedagogía Escolar dominante, lo que significaba que la escuela y la educación infantil constituían la principal referencia pedagógica para las enseñanzas de las personas adultas; una situación que en parte se modifica con 
la LOGSE con cambios en esta dirección, pero insuficientes y poco ambiciosos. c) En la medida en que las leyes se han centrado casi exclusivamente en regular las enseñanzas del sistema educativo formal y oficial, han dejado fuera buena parte de la educación no formal e informal que realizan las personas adultas, impidiendo el desarrollo socioeducativo de este campo de la educación, con consecuencias sociales negativas para el desarrollo y el bienestar ciudadano.

No obstante, si tenemos en cuenta las aportaciones de diferentes autores e investigadores, basadas en no pocas realizaciones educativas, lo que vemos es todo un sector educativo, la educación de personas adultas, que aspira y avanza afirmando su identidad en una triple dirección: a) Constituye una oferta educativa propia y específica, con valor en sí misma, basada en el principio de educación permanente; b) Se trata de un tipo de educación social en la que el desarrollo personal es inseparable de la mejora del tejido social, razón por la cual precisa un tratamiento específico y diferenciado, cuyo marco teórico y metodológico de referencia es la Pedagogía Social; c) Como tal oferta de educación social, debe ser contemplada en el contexto de la educación universal de un país, lo que significa que las leyes educativas deben concebir la educación en términos más amplios, incluyendo todas las oportunidades de educación formal, no formal e informal.

La pregunta, por tanto, es ¿por qué esta disonancia? ¿Por qué esta diferencia entre lo que dicen las leyes y normas y las aportaciones teóricas que, además, se basan en determinadas prácticas?

Desde nuestro punto de vista, la razón es que no ha habido política educativa alguna en materia de educación de adultos. Si entendemos por política educativa las decisiones y acciones de los poderes públicos que, basadas en valores y metas compartidas, orientan, a través de leyes y directrices, la acción educativa de una sociedad, esto no se ha aplicado al sector de la educación de adultos. En las leyes estudiadas no hemos visto diseño alguno, cierta lógica educativa o una planificación estratégica, sino más bien desconocimiento, abandono, falta de interés, cierto caos, o no saber qué hacer, porque en todo momento las prioridades han sido otras. Y todo ello porque, simplemente, la concepción pedagógica que lo justifica no ha trascendido a la sociedad, ni se ha desplegado por las mesas de los legisladores, o, dicho de otra manera, no ha conseguido traspasar los muros de la Academia.

En el pasado esto podría explicarse teniendo en cuenta el escaso desarrollo práctico y la debilidad teórica de este sector de la educación. Pero actualmente las cosas han cambiado: por un lado, la educación de personas adultas ha experimentado en las últimas décadas un incuestionable crecimiento y desarrollo, con efectos personales y comunitarios que no acaban de ser identificados y reconocidos por la sociedad en la que vivimos; $y$, por otra parte, en el seno de las universidades se han creado grupos de investigación y de estudio que han conseguido identificar unas señas de identidad para este campo de la educación en términos de educación social y en el marco de la Pedagogía Social, cuyo caudal pedagógico tampoco ha conseguido ser identificado y reconocido por el resto de la comunidad académica.

Que la educación de personas adultas ha crecido no hay duda, y menos aún, sobre la función social que cumple. Actualmente ya tenemos estadísticas con cifras de participación que van desde el $30 \%$ hasta el $50 \%$ según los países; un hecho inédito que comienza a conocerse desde mitad del siglo $\mathrm{XX}$, consistente en que cada vez es mayor el número de personas adultas que se forma. De tal manera, que se ha invertido la tendencia tradicional, pues el volumen de población adulta que participa en procesos de educación hoy es mayor que la totalidad de la población infantil y adolescente escolarizada (Bélanger, 1999). En España, según la encuesta sobre Participación de la Población Adulta en Actividades de Aprendizaje (EADA, 2016), vemos que la población, entre 18 y 64 años, que ha realizado actividades de educación formal alcanza los 4.715.973, siendo más de un millón trescientos mil los que tienen entre 35 y 64 años; también se aportan datos de las personas entre 18 y 64 años que han realizado actividades de educación no formal en el mismo año 2016: en total son 11.822.954, en cuyo caso los que tienen entre 35 y 64 años superan la cifra de siete millones y medio.

El problema es que no se conoce la dimensión y el alcance de estos datos y mucho menos los efectos personales, culturales, económicos, sociales y políticos que tiene esta realidad. Cuestiones como la salud, el bienestar, el empleo, el mercado de trabajo, la vida social, la equidad, la participación 
comunitaria, etc. mejoran a través de la educación de adultos, como se reconoce en este informe de la UNESCO (2010): "El aprendizaje empodera a los adultos al darles los conocimientos y las competencias para mejorar sus vidas. Pero también beneficia a sus familias, comunidades y sociedades. La educación de adultos desempeña un papel importante en la lucha contra la pobreza, la mejora de la salud y la nutrición, así como en la promoción de prácticas sostenibles del medio ambiente" (pp. 8). Un mensaje este que se quiere hacer llegar a los responsables políticos: "los actores sociales encontrarán pruebas y argumentos convincentes para sostener cómo el aprendizaje y la educación de adultos promueven el desarrollo sostenible, sociedades más saludables, mejores trabajos y una ciudadanía más activa" (UNESCO, 2017, pp. 9). Por todo ello, las políticas, estrategias y presupuestos, basadas en el aprendizaje permanente, constan como uno de los objetivos clave de Agenda 2030 para el desarrollo sostenible (ONU, 2015, pp.16).

Por otra parte, podemos afirmar que, a diferencia también del pasado, las universidades, cumpliendo con su función investigadora y docente, han conseguido superar buena parte del escaso desarrollo y debilidad teórica que tradicionalmente ha padecido este campo de la educación. Sobre todo, a partir de 1991, cuando se aprueba el título universitario de Educación Social, en el que se define la "educación de adultos" como uno de sus ámbitos de intervención, consolidando así la Pedagogía Social en más de 22 universidades españolas (Caride Gómez, 2009). Pero nuevamente sorprende que la comunidad académica de los pedagogos, los historiadores de la educación, los didactas y otros expertos en la materia no hayan percibido la riqueza educativa, social y política que ha ido ganando la educación de las personas adultas con el paso del tiempo. Desde nuestro punto de vista, ello es debido a lo que ya hemos comentado anteriormente: que la Pedagogía se ha tornado "Pedagogía Escolar", articulada en base a dos variables clave: los niños, como destinatarios principales de la educación, y la escuela, como espacio privilegiado de la acción docente; dos variables que, además, han terminado fortaleciendo la identificación entre educación y sistema educativo formal y oficial, en el que los infantoadolescentes representan casi exclusivamente todo el alumnado.

Tal miopía ha conducido a que cuando se habla de educación en la familia, en la calle, en los medios de comunicación, en el parlamento y en otros foros de debate, en realidad no estemos hablando de la educación de toda la sociedad, del universo educativo, toda vez que se deja fuera buena parte de la educación que tienen y realizan las personas adultas. Una posición que no contempla la totalidad de la educación sino parte de ella, como si determinados problemas educativos (como la intolerancia, la ignorancia, la manipulación, las desigualdades, la violencia de género, el calentamiento global, las derivas políticas autoritarias, las fake news, etc.) no afectarán también a las personas adultas.

Como ya se ha dicho anteriormente, de este modo se da la espalda (o peor, se ignora o desprecia) a los efectos educativos y culturales, económicos y sociales, políticos y comunitarios que se generan en una sociedad cuyas personas adultas han optado por aprender a lo largo de su vida, mejorando al mismo tiempo sus relaciones sociales, y todo ello, además, sin que necesariamente tenga que ver con la perspectiva de un título oficial o de mejorar en el trabajo. En el fondo, lo que les mueve es el convencimiento de que el aprendizaje no tiene edad y la posibilidad de compartir la curiosidad y el interés por el conocimiento, debido, entre otras razones, a determinados cambios personales y sociales que afectan sobre todo a las personas adultas: el incremento de la esperanza de vida, el hecho de que las personas mayores viven muchos años y lo hacen saludablemente, y que se trata de un colectivo cada vez más numeroso.

Por ende, teniendo en cuenta que, de los tres grandes desafíos que han condicionado la evolución de la educación de las personas adultas a lo largo de su historia, se ha conseguido superar el primero (oferta educativa específica y con valor en sí misma), planteamos para el debate dos propuestas para discutir su viabilidad, si hablamos de una política educativa en el campo de la educación de adultos: que las leyes de educación aborden el universo educativo y que la educación de adultos cuente con un tratamiento diferenciado en dicho universo. 
Por un lado, se plantea que las leyes educativas, partiendo de una concepción amplia de la educación, aborden el universo educativo, concebido como la totalidad de los procesos de educación que tienen lugar en una sociedad, sea cual sea el tipo de educación (formal, no formal e informal) y cualquiera que sea la edad de los participantes; hablamos por tanto de un amplio escenario de la educación (Delors et al., 1996; Comisión Europea, 2000; Vázquez Gómez, 2002; Colom Cañellas, 2005) que, basado en el principio de educación permanente, contemple la realidad de la educación en la doble dirección temporal (a lo largo de la vida) y espacial (a lo ancho de la vida); universo educativo, en fin, que debe ser promovido y financiado por todas las autoridades e instituciones del país. Lo cual no impide, por supuesto, que las autoridades educativas, de acuerdo con la Constitución española, cumplan con su obligación de regular lo relacionado con las enseñanzas formales, que conducen a las titulaciones oficiales del sistema educativo, reconocidas y acreditadas por el Estado.

Por otra parte, se propone que en dicho universo educativo coexistan varios subsistemas con tratamientos pedagógicos, organizativos y administrativos diferentes. Uno de tales subsistemas podría ser el de la educación de personas adultas con dos grandes áreas: por un lado, la educación formal que conduce a las titulaciones oficiales, que debe ser regulada legalmente, pero con un tratamiento diferente al resto de las enseñanzas (currículo y organización propia), de acuerdo con pautas teóricas y metodológicas propias de la Pedagogía Social, y teniendo en cuenta, además, las características psicológicas, culturales y sociales de la población adulta y sus comunidades; y, en segundo lugar, la educación no formal e informal, que, dadas sus características, no requieren regulación legal alguna (en los mismos términos que las enseñanzas formales), pero sí promoción, financiación, seguimiento científico, formación de los profesionales, apoyo de experiencias y proyectos, sistemas de calidad, gestión de centros, etc. Al mismo tiempo, no podemos hablar del subsistema de educación de adultos con las dos áreas citadas, sin regular legalmente las necesarias pasarelas entre educación formal y educación no formal e informal, mediante sistemas de acreditación; solo de este modo las personas adultas podrán ejercer su derecho a que las competencias y aprendizajes que han adquirido a través de la experiencia y/o por medio de las enseñanzas no formales puedan ser reconocidas y acreditadas en términos de enseñanza formal.

\section{Referencias}

Bélanger, P. (1999). La amenaza y la promesa de una sociedad que reflexiona. Nuevo entorno político de la educación de adultos. Educación de Adultos y Desarrollo, 52, 193-211.

Cabello Martínez, M. J. (2002). Educación Permanente y Educación Social: controversias y compromisos. Aljibe.

Camacho Herrera, A. (1999). La Educación de Adultos y el Desarrollo Comunitario. Cuestiones Pedagógicas, 14, 197-215. https://bit.ly/2CbrzTV

Caride Gómez, J. A. (2009). Elogio de la Pedagogía Social: acerca de los viejos y nuevos desafíos de la educación social. Revista de Educação Pública, 18(38), 449-468. https://bit.ly/2N2ANEe

Colom Cañellas, A. (2005). Continuidad y complementariedad entre la educación formal y no formal. Revista de Educación, 338, 9-22. https://bit.ly/30LSzmX

Comisión Europea (2000, 30 de Octubre). Memorándum sobre el Aprendizaje Permanente. Bruselas: Comisión de las Comunidades Europeas. https://bit.ly/30Oh1nR

Consejería de Educación, Cultura y Deportes (1992). Diseño curricular base para la Educación de Adultos de Canarias. Estructura y elementos del diseño (Primer borrador). Las Palmas de Gran Canaria: Dirección General de Promoción Educativa, Gobierno de Canarias. 
Hernández, N. y Llop Chulvi, M. (1995). La formación básica de personas adultas. ¿Retórica o dialéctica? Nau Llibres.

Delgado Granados, P. (2005). Las primeras experiencias de educación popular y regeneración social en jóvenes. XXI. Revista de Educación, 7, 73-79. https://bit.ly/3fsyaaG

Delors, J. (1996). La educación encierra un tesoro. Santillana-UNESCO.

EADA (2016). Encuesta sobre Participación de la Población Adulta en Actividades de Aprendizaje. Instituto Nacional de Estadística. https://bit.ly/30LRv2C

Flecha, R. (1990). Educación de las personas adultas. Propuesta para los años noventa. El Roure.

Flecha, R. López Palma, F y Saco Coya, R. (1988). Dos siglos de educación de adultos. El Roure.

Formariz Poza, A. (1995). Educación social y educación de personas adultas. Educación Social. Revista de intervención socioeducativa, 1, 51-61.

García Carrasco, J. (Coord.) (1997). Educación de adultos. Ariel.

Gobierno de España (1857, 10 de septiembre). Ley de Instrucción Pública. Boletín Oficial del Estado 1710. https://bit.ly/3kDhsrO

Gobierno de España (1945, 17 de julio). Ley de Educación Primaria. Boletín Oficial del Estado 199. https://bit.ly/3kKQQ8t

Gobierno de España (1970, 6 de agosto). Ley General de Educación y Financiamiento de la Reforma Educativa. Boletín Oficial del Estado 187. https://bit.ly/2FTw5bF

Gobierno de España (1974, 14 de febrero). Orden de Orientaciones Pedagógicas para la Educación Permanente de Adultos, a nivel de Educación General Básica. Boletín Oficial del Estado 55. https://bit.ly/2RSWGZ4

Gobierno de España (1990, 3 de octubre). Ley de Ordenación General del Sistema Educativo. Boletín Oficial del Estado 238. https://bit.ly/35Zpasd

Gobierno de España (2002, 19 de junio). Ley de las Cualificaciones y de la Formación Profesional. Boletín Oficial del Estado 147. https://bit.ly/3cqDQSc

Gómez R. de Castro, F. (1995). La educación de las Personas Adultas a lo largo de la historia. Perspectiva nacional e internacional. En F. Sanz Fernández (Coord.), La formación en Educación de Personas Adultas (pp.157-200). Tomo I. UNED.

Guereña, J. L. (1992). Los orígenes de la educación de adultos en la España contemporánea. En A. Escolano (Dir.), Leer y escribir en España. Doscientos años de alfabetización (pp.281-307). Pirámide.

Guereña, J. L. y Tiana Ferrer, A. (1994). La educación popular. En J.L. Guereña, J. Ruiz Berrido. y A. Tiana Ferrer (Eds.), Historia de la Educación en la España Contemporánea. Diez años de investigación (pp. 141-171). CIDE/Centro de Publicaciones del Ministerio de Educación y Ciencia.

Jabonero Blanco, M., Nieves Cazorla, M. R. y Ruano Morcuende, M.I. (1997). Educación de personas adultas: un modelo de futuro. La Muralla.

Lancho Prudenciano, J. (2005). La Educación de Adultos en la España autonómica. UNED. https://bit. ly/3mlkw7T 
Lucio-Villegas Ramos, E. (1993). La investigación participativa en educación de personas adultas. La construcción de un saber colectivo. CAPP-Kronos.

Marzo, A. y Figueras, J. M. (1990). Educación de adultos. Situación actual y perspectivas. Horsori.

MEC (1986). Libro Blanco de la Educación de Adultos en España. Ministerio de Educación y Ciencia.

MEC (1989). Libro Blanco para la Reforma del Sistema Educativo. Ministerio de Educación y Ciencia.

MEC y CCAA (1993). Conclusiones de los grupos de trabajo de las Jornadas Técnicas para el Desarrollo de la LOGSE en materia de Educación de Personas Adultas. Materiales para la Educación de Adultos, 5, 16-19.

MEC y CCAA (1995). Acuerdos sobre los elementos comunes a todo el Estado para la elaboración de un diseño curricular específico de la Formación Básica de las personas adultas. Materiales para la Educación de Adultos, 6-7, 8-18.

Medina Fernández, O. (1997). Modelos de Educación de Personas Adultas. El Roure.

Medina Fernández, O. (2000). Especificidad de la Educación de Adultos. Bases psicopedagógicas y señas de identidad. Educación XX1, 3, 91-140. https://doi.org/10.5944/educxx1.3.1.406

Mezirow, J. (1994). Transformaciones en la educación y aprendizaje de adultos. En J. Sáez Carreras y F. Palazón Romero (Coords.), La Educación de Adultos: ¿una nueva profesión? (pp. 181-187). Nau Llibres.

Moreno Martínez, P. L. (1992a). La Ley General de Educación y la educación de adultos. Revista de Educación (núm. extr.), 109-130. https://bit.ly/37z1PMY

Moreno Martínez, P. L. (1992b). De la alfabetización a la educación de adultos. En A. Escolano (dir.), Leer y escribir en España. Doscientos años de alfabetización (pp. 111-140). Pirámide.

Moreno Martínez, P. L. y Viñao Frago, A. (1997). La Educación de Adultos en España (siglos XIX y XX): Historia de una realidad cambiante y multiforme. En J. García Carrasco (Coord.), Educación de adultos (pp. 23-46). Ariel.

ONU (2015, 21 de octubre). Agenda 2030 para el Desarrollo Sostenible. Resolución aprobada para la Asamblea General el 25 de septiembre de 2015. https://bit.ly/3e620Se

Quintana Cabanas, J. M. (Coord.) (1986). Investigación Participativa Educación de Adultos. Narcea.

Requejo Osorio, A. (1992). La Educación de Adultos y la reforma del sistema educativo. Materiales para la Educación de Adultos, 3-4, 10-20.

Requejo Osorio, A. (2003). Educación permanente y educación de adultos: intervención socioeducativa en la edad adulta. Ariel.

Sáez Carreras, J. (1998). La formación de personas adultas: ¿para una sociedad pasiva o la consecución de personas críticas? En J. Sáez Carreras y A. Escarbajal de Haro (Coords.), La Educación de personas adultos. En defensa de la reflexividad crítica (pp. 79-114). Amarú.

Sáez Carreras, J. (Coord.) (2007). Pedagogía Social y Educación Social. Pearson Prentice Hall.

Sanz Fernández, F. (Coord.) (2002). La educación de las personas adultas entre dos siglos: historia pasada y desafíos de futuro. Ministerio de Educación, Cultura y Deportes y UNED. 
Sanz Fernández, F. (2006). El aprendizaje fuera de la escuela. Tradición del pasado y desafío para el futuro. Ediciones Académicas.

Sarrate Capdevila, M. L. y Pérez de Guzmán Puya, M. V. (2005). Educación de Personas Adultas: situación actual y propuestas de futuro. Revista de Educación, 336, 41-57. https://bit.ly/2UNcQ86

Sebastián Vicente, A. (2001). Educación de adultos, democracia y participación ciudadana. En F. López-Noguero, R. Cruz-Díaz y R. Beltrán Ruiz (Coords.), Formación permanente: tendencias actuales. Asociación Educa-Acción.

Tiana Ferrer, A. (1991). La educación de adultos en el siglo XIX: los primeros pasos hacia la constitución de un nuevo ámbito educativo. Revista de Educación, 294, 7-26. https://bit.ly/37yaM9h

Tiana Ferrer, A, y Sanz Fernández, F. (Coords.) (2003). Génesis y situación de la educación social en Europa. Madrid: UNED. https://bit.ly/2Hmlsyx

Trilla Bernet, J. (2002). El universo de la Educación Social. En M. Romans, A. Petrus, A. y J. Trilla. De profesión: educador(a) social (pp. 13-59). Paidós.

UNESCO. (2010). Global Report on Adult Learning and Education. Instituto de la UNESCO para el Aprendizaje a lo Largo de la Vida. https://bit.ly/30JlvvX

UNESCO (2017). Tercer Informe Mundial sobre el Aprendizaje y la Educación de Adultos. Instituto de la UNESCO para el Aprendizaje a lo Largo de la Vida. https://bit.ly/2MXsaL7

Vázquez Gómez, G. (2002). El sistema educativo ante la educación de calidad para todos a lo largo y ancho de la vida. Revista de Educación (núm. extr.), 39-57. https://bit.ly/2AGdL3b

Viladot, G. y Romans, M. (1988). La Educación de Adultos. Laia.

Viñao Frago, A. y Moreno Martínez, P. L. (2003). La periferia del sistema educativo. Cuadernos de Pedagogía, 326, 76-80. 